\title{
Tangence
}

\section{Pratiques utopistes et discours muséologique dans l'écriture de Nicole Brossard et de Cristina Peri Rossi}

\section{Claudine Potvin}

Numéro 47, mars 1995

Écritures au féminin : le genre marqué

URI : https://id.erudit.org/iderudit/025850ar

DOI : https://doi.org/10.7202/025850ar

Aller au sommaire du numéro

Éditeur(s)

Tangence

ISSN

0226-9554 (imprimé)

1710-0305 (numérique)

Découvrir la revue

Citer cet article

Potvin, C. (1995). Pratiques utopistes et discours muséologique dans l'écriture de Nicole Brossard et de Cristina Peri Rossi. Tangence, (47), 42-55.

https://doi.org/10.7202/025850ar d'utilisation que vous pouvez consulter en ligne. 


\section{Pratiques utopistes et discours muséologique dans l'écriture de Nicole Brossard et de Cristina Peri Rossi}

\section{Claudine Potvin, Université de l'Alberta}

Je propose ici une brève analyse du discours muséographique dans un récit de Nicole Brossard (Picture Theory) et un roman de Cristina Peri Rossi (La nave de los locos) à partir d'une discussion théorique et pratique de la figure d'utopie dans ces mêmes textes ${ }^{1}$. Deux livres différents, deux auteures d'origine fort éloignée (le Québec et l'Uruguay) et malgré tout, deux écritures qui se rejoignent: éclatement de la prose, fragmentation du récit en mille éclats, en autant d'histoires, déconstruction du genre narratif, personnages en transition, en état de voyage, polysémie, rapport ludique à la langue, préoccupations féministes, fictions lesbiennes.

Picture Theory raconte sous forme poétique l'élaboration d'un livre de femme composé par une narratrice qui mêle les mots à ses amours et au langage de quelques filles dites studieuses. et radicales en train de repenser l'abstraction. Peri Rossi met également en scène à sa manière, à travers les multiples voyages de son protagoniste, une exploration du langage et de la nature humaine. L'utopie s'articule ici en partie autour des motifs du musée, de la ville et de la frontière qui les traverse. En effet, ou bien la narratrice, le narrateur, y circulent inscrivant le geste, le mouvement, la séduction, "la " continent des femmes afin d'y projeter une forme d'utopie intégrale; ou bien ils choisissent la forme du musée comme lieu, limite, toile de fond, allégorie, décor, cadre, échange, regard, introspection.

Foucault considère le musée comme une des hétéropies privilégiées de la société occidentale, c'est-à-dire comme une utopie réalisée correspondant à des emplacements spécifiques, désignés,

1 Nicole Brossard, Picture Tbeory, Montréal, l'Hexagone, 1982; Cristina Peri Rossi, La nave de los locos, Barcelona, Seix Barral, 1984. Pour toute référence à ces textes, j'indiquerai dorénavant la page entre parenthèses à la suite de la citation. 
44

reflétés ou réfléchis par tous les autres emplacements avec lesquels ils sont en rapport direct ${ }^{2}$. Frontière entre l'objet et sa représentation, reflet, projection, le musée surgit dans l'imaginaire de Brossard et de Peri Rossi sous forme de amonument . historique, archéologique, esthétique, politique, simultanément public et privé. "Original", il exige toutefois une fiction qui lui serve de commentaire et le justifie comme univers de représentation. Le corps-musée de la ville patriarcale y renvoie par ailleurs à une scène de performance, trans(e)/formation, pur "propos de civilisation" (p. 41, 207), écrit Brossard, pur spectacle. L'éclat du musée, de l'art, de la création, de la collection d'objets ou de mots, s'opère dans le récit pour que la matière ainsi éclatée s'assemble dans le livre.

Musée/mausolée; exposition d'objets sans valeur et/ou, au contraire, consacrés par un discours critique absolu. Or, inscrite dans le texte, prétexte artificiel ou objet de discours, l'expérience muséographique (installation, évènement, exposition, lecture, conférence, visite) organise l'utopie d'un savoir autre qui pose la problématique de l'insertion de la parole des femmes dans l'histoire et dans l'histoire de l'Art. Déterritorialisation, le langage, chez Brossard et Peri Rossi, représenterait alors une frontière ouverte qui nie l'unicité et tend à déplacer les traces soi-disant "Originales", "authentiques", les espaces ethnoculturels, et à permettre des assemblages et des agencements différents, des aménagements complexes, l'altérité, le paradoxe utopique lui-même.

La pratique de l'utopie est de fait souvent ambivalente et paradoxale; dans son rapport à l'écriture, elle engendre le dépassement ou la mutation des ruptures et des totalités, ce que Pourbaix nomme "la totalité de la rupture" et "la rupture de la totalité". Le critique souligne que l'absence de lieu qu'est l'utopie interroge le politique et que la pulsion d'utopie détourne précisément les exigences sociales "en définissant un niveau interne, individuel, où se joue une véritable exigence où se produisent les véritables mythologies qui viendront ensuite se manifester au niveau plus global du social ${ }^{3}$. Toute appropriation collective du

2 Michel Foucault, - Des espaces autres •, conférence présentée à Paris le 14 mars 1967 et publiée dans A.M.C. Rerue d'architecture (1967). Cité dans Lucía Invernizzi, . Entre el tapiz de la expulsión del paraíso y el tapiz de la creación del viaje a bordo de la nave de los locos de Cristina Peri Rossi., Revista chilena de Literatura, $\mathrm{n}^{\circ} 30$, novembre 1987, p. 37.

3 Joël Pourbaix, - Paul Chamberland: La posture utopiste., Jacques Pelletier (dir.), L'auant-garde culturelle et littéraire des années 70 au Québec, Montréal, 
monde et de soi efface jusqu'à un certain point l'immuabilité des choses et de l'être humain ${ }^{4}$.

Fantasme textuel/sexuel, intime ou collectif, le musée représenté ou reproduit dans ces fictions tient un discours socioculturel sur ce qui y loge ou n'y loge pas. La série, la suite (de la galerie, de la zone urbaine ou autre), contient la bordure et la transgresse ; la parodie y signale et abolit parallèlement la frontière. En ce sens, l'écriture de ces écrivaines cherche à retrouver tous les sens, recréer le show permanent, repenser l'utopie en termes postmodernes. Comme dans le musée, le contexte n'y est jamais donné mais produit.

Au départ, le musée renvoie à la sélection, la collection d'objets qui recréent ou cherchent à créer l'illusion de la cohérence: "For the museum seemed to be equally a space of exclusions and confinement" ${ }^{5}$. Le musée classique (symbolisé ici par une tapisserie médiévale nommée Tapiz de la creación chez Peri Rossi), physiquement composé d'une enfilade de chambres/ textes, assure un effet de continuum, ce que l'écrit vient briser à plus d'un titre puisque les mots ne s'enchaînent pas nécessairement ou dans tous les cas, à l'envers, par brisures, par détachements, par fragments. De fait, il convient de reprendre ici la définition du musée que donne Eilean Hooper-Greenhill au tout début de son ouvrage Museums and the Shaping of Knowledge: "Museums, remarque-t-elle, are no longer built in the image of that nationalistic temple of culture, the British Museum. Today, almost anything may turn out to be a museum, and museums can be found in farms, boats, coal mines, warehouses, prisons, castles, or cottages * 6 . Roger Silverstone, dans son article intitule "The

Université du Québec à Montréal, . Cahiers du département d'études littéraires -, 1986 , p. $86-87$.

4 Pour la notion d'utopie, voir mon article sur Babel, prise deux de Francine Nö̈l, - De l'Éden à Babel : écrire l'utopie , dont je reprends quelques éléments théoriques ici. Paru dans Voix et images, vol. XVIII, n.2, hiver 1993, p. 287-303.

5 Douglas Crimp, - The Postmodern Museum •, Parachute, mars-mai 1967, p. 62. Traduction: - Car le musée semble être à la fois un espace d'exclusion et de confinement.

6 Eilean Hooper-Greenhill, Museums and the Shaping of Knowledge, London, Routledge, 1992, p. 1. Traduction: * La construction des musées ne correspond plus à l'élaboration d'un temple national de la culture comme le Britisb Museum par exemple. De nos jours, tout est susceptible de devenir musée; 
46

medium is the museum: on objects and logics in times and spaces *, va dans le même sens et ajoute que:

The museum is no longer, if ever it was, an institution which can be understood in its own terms as innocently engaged in the processes of the collection, conservation, classification and display of objects. On the contrary, it is one of the many components in a complex array of cultural and leisure industries, no longer certain of its role, no longer secure in its identity, no longer isolated from political and economic pressures or from the explosion of images and meanings which are, arguably, transforming our relationships in contemporary society to time, space and reality. ${ }^{7}$

Lauro Zavala maintient de son côté que le musée est avant tout un espace de l'excès. Il accumule et exhibe l'excès social de l'information, du temps, du quotidien: ex/centrique, asymétrique, il s'approprie l'espace de la rue, de l'histoire, de la culture, de l'expérience personnelle, de l'art, etc. ${ }^{8}$

Dans le roman de Nicole Brossard, le musée se trouve à peine inscrit au cour du récit: cinq mentions en tout, quelques références sous formes allusives (tableau, toile, stèle). Or, l'expérience muséographique, toute dans le désir de celle qui visite, de celle qui circule et "entre au Musée" (p. 45, 46, 56) comme on entre au Hilton, de la narratrice/écrivaine qui s'installe dans la chambre, visionne le corps d'une femme, la perception du musée donc et des objets exposés à la théorie du réel est entièrement contenue dans le titre du livre, Picture Theory. Picture, soit écran,

ainsi une ferme, un bateau, une mine de charbon, un entrepôt, une prison, un château et même une maison peuvent tour à tour abriter un musée..

7 Roger Silverstone, The medium is the museum: on objects and logics in times and spaces", Towards the Museum of the Future. New European Perspectives, Roger Miles \& Lauro Zavala (dir.), Londres, Routledge, 1994, p. 161. Traduction: "Le musée n'est plus, mais l'a-t-il déjà été, une institution innocente, engagée à partir de ses propres critères dans un processus de collection, de conservation, de classification et d'exposition d'objets. Au contraire, il constitue une composante parmi d'autres d'une industrie culturelle dorénavant incertaine de son rôle et de son identité, soumise aux pressions économiques et politiques et à une explosion permanente d'images et de significations qui transforment notre rapport au temps, à l'espace et à la réalité dans la société contemporaine. .

8 Voir Lauro Zavala, Ma. de la Paz Silva, J. Francisco Villaseñor, Posibilidades y límites de la comunicación museográfica, Mexico, Universidad Nacional Autonóma de México 1993, p. 31. 
tableau, toile/scène blanche sur laquelle se projette l'écriture de la narratrice, peintures, reproductions; copies, enlevées au cadre institutionnel; l'art de voir et d'écrire se pratique dans la rue, hors-les-murs, comme à Beaubourg, éclaté: "L'éclat du musée, ce que la fiction donne à lire, répète l'auteure. Mille fragments retombent sur mes épaules. De la matière partout: pièces d'identité : notes, lipstick, miroir, condom, clés, argent, mille fragments s'assemblent sous vos yeux dans le musée, dans le livre, il faut les voir venir" (p. 130).

Le livre fait musée, provoque un effet "tableau " non seulement parce qu'une forme d'image y interpelle la lectrice ou parce que l'art s'y trouve inscrit dans le texte (voir l'hologramme de la dernière partie), mais parce que l'écriture y est perméable à un certain système de représentation qu'elle encode et décode. Dans ce contexte, les termes musée, art, toile, tableau sont pris ici dans un sens large, écriture et art s'incluant l'un l'autre.

Le musée, ce sera donc par extension un espace utopique ouvert semblable au livre, qui s'arpente, se marche, se parcourt de long en large, se transforme à chaque lecture, à la mesure des pas, des arrêts, des doutes, des angoisses, des soupirs, des regards de la spectatrice ou de l'écrivante, des reconnaissances. Le musée, c'est à la fois le canon, le classique, le connu, l'invitation au voyage, le naif, la subversion, l'étonnant, le moderne, Joyce, Gertrude Stein. Photographies, sculptures, peintures, montages, collages, installations, cadres, objets, corps, matières, matériaux, extérieurs, intérieurs, fenêtres, etc., le visuel accroche, cherche une forme d'émotion. Si Michèle Vallée, la protagoniste de Picture Theory * connaissait peu de l'émotion. (p. 165), Claire Dérive, l'amante, et sa sœur Florence, elles, pensent le rêve, " dérivent" au-delà d'une clarté visible sur le dos de la mer, emportant avec elles un flot de femmes/flammes ayant recours à l'abstraction.

Il faut entendre le musée dans le même sens chez Peri Rossi quoique chez cette dernière le motif du musée, imaginaire ou réel, spatial ou temporel, ancien et moderne, tend à envahir le récit. Dans le roman retenu ici, La nave de los locos, l'intertexte renvoie à Érasme, Brueghel, Bosch en ce qui concerne la représentation de "la nef des fous", à la tradition iconographique de la roue de fortune ainsi qu'à une toile inventée recréant une autre traversée allégorique au cours de laquelle on élimine ces marginaux que 
représentent les fous au $\mathrm{Xv}^{e}$ siècle (motif repris dans la reproduction de Jan Bale sous le signe de l'ordre cette fois), l'écran cinématographique (musée pornographique), enfin une tapisserie médiévale. Je ne m'occuperai ici que des onze passages descriptifs dans lesquels l'auteure présente cette tapisserie appelée le Tapiz de la creación qui sert de contrepoint à la topique de la nef des fous. Le Tapiz, ouvrage médiéval accroché aux murs de la Cathédrale de Gérone, exemplifie "la religiosidad medieval capaz de construir un mundo perfectamente concéntrico y ordenado" (p. 20) 9 . Métaphore de la création du monde telle que la présente la Genèse et symbole de l'harmonie universelle, l'oeuvre d'art, par extension le musée, s'intègre ici aux voyages du personnage central, Equis, visiteur et spectateur distant avant tout, et forme la toile de fond sur laquelle se tisse et se dessine le texte du roman. Voyager, visiter un musée, revient à marquer et effacer des frontières, couper et fabriquer des liens, confondre et identifier des lignes, s'éloigner de la rive et s'approcher d'un autre lieu, mélanger et déchiffrer des cartes, des routes, des cadres, dé(limiter) ses territoires, reconnaître et oublier des horizons, déplacer le centre, nommer, se trouver et se perdre.

Par opposition au contexte de la folie, incontrôlable et incontrolé, le Tapiz de la creación propose un univers à prime abord parfaitement géométrique, symétrique, vérifiable, ordonné. "Todo en el tapiz, responde a la intención de que el hombre que mira - espejo del hombre representado con hilos de colores participa de la creación... y para que sin salirse de los límites de la tela, esté en el centro miśmo de la creación, no por ello alejado de los bordes o extremos* (p. 20) ${ }^{10}$. Bien que jamais totalement éloigné de la périphérie, le spectateur se voit ainsi pris dans la fable puisqu'on le force en son centre. L'ambivalence entre l'attraction/séduction presque fatale face au tableau de la création du monde et le rejet d'une vision unique, rationnelle et intégrée de l'univers, se joue dans le regard et la position de Equis, luimême crẹ́ation/objet d'art hors-contexte, en marge, en retrait, isolé, sous la ligne blanche qui sépare le récit du reste de la page.

9 Traduction: e[...] la religiosité médiévale capable de penser un monde parfaitemente concentrique et ordonné.

10 Traduction: - La tapisserie en entier permet que l'bomme qui regarde reflet de l'homme représenté dans les fils de couleur - participe de la création... et que, sans sortir des limites de la toile, il se situe au centre même de la création, sans pour autant s'éloigner du bord ou des extrémités.: 
C'est d'ailleurs dans une note en bas de page que l'on apprend qu'il était certes fasciné par la scène mais non pas nostalgique.

Le discours essentiellement euphorique que la première lecture de la tapisserie autorise se désarticule et se renverse au fur et à mesure que le cadre laisse transparaître une dimension moins uniforme: le bonheur idyllique (la faune, la flore, le Saint-Esprit, le soleil, la lune, Adam et Ève, le paradis terrestre), fait place au chaos et au désordre (mutilation et fragmentation de l'étoffe, condamnation des créatures enfermées dans la toile, surveillance et contrôle d'une seule autorité). Le musée, la toile, le texte organisent le monde en images et en perceptions, fabriquant et déconstruisant des utopies en fonction d'un système idéologique de pensée et de reconnaissance ou de transgresssion des valeurs établies. Néanmoins, lieux limites où se concentre la charge utopique maximale, où tout est encore possible; lieux de frontière, de l'entre-deux, de la séduction et du désir, de la peur et de l'inquiétude aussi.

Selon Louis Marin, la fonction de l'utopie est transgressive: c'est une critique idéologique de l'idéologie ${ }^{11}$. Darko Suvin, pour sa part, ajoute à cette idée le concept de jeu "mené par quelqu'un qui a le sens des possibles autres que ceux de la nature, surtout des possibles parallèles " ${ }^{12}$. Pour Barbara Godard qui commente les travaux de ces deux critiques, l'utopie sousentend l'idée du double, soit *la projection métaphorique de la réalité "ailleurs" et le déplacement métonymique des structures de la société contemporaine. Selon Godard, l'utopie interrompt de la sorte le discours idéologique, d'où la production de nouvelles structures susceptibles d'opérer des transformations sociales ${ }^{13}$. Les écrits utopistes ne construisent pas toujours une fiction utopique mais réalisent quelquefois une fiction du réel basée sur une représentation des faits.

La nave de los locos et Picture Theory élaborent chacun à sa manière de nouvelles utopies féministes construites sur une

11 Voir Louis Marin, Utopics: Spatial Play, traduit par A. Vollrath, Atlantic Highlands, N. J., Humanities Press Inc., 1984. Titre original: Utopiques: Jeux d'espace.

12 Darko Suvin, Metamorphoses of Science Fiction: The Poetics and History of a Genre, New Haven, Yale University Press, 1979, p. 53.

13 Voir Barbara Godard, .En mémoire de l'avenir : les stratégies de transformation dans la narration de Jovette Marchessault, Voix et images, vol. XVII, $n^{\circ} 1$, automne 1991, p. 105-106. 
abstraction des lieux et des personnages, un réarrangement de la fiction et de la grammaire des sexes, un déplacement de la réalité. Pour reprendre à nouveau Godard, ajoutons que *la pratique de l'utopie avance un projet féministe de critique et de transformation des codes du genre romanesque en autant qu'ils se révèlent être des codes du discours sexué" 14 . Ces nouvelles utopies ne sont pas des endroits fixes ou statiques car, comme le signale Northrop Frye, "they would not be rational cities evolved by a philosopher's dialectic: They would be rooted in the body as well as in the mind, in the unconscious as well as in the conscious, in forests and deserts as well as in highways and buildings, in bed as well as in the symposium ${ }^{15}$. Selon Frye, l'utopie n'est pas un lieu puisque à la question qu'il pose "où est l'utopie", il répond "nulle part", ce qui revient à dire "ici " ou partout peut-être.

Dans Picture Theory, le corps traverse l'espace de la ville car il s'agit d'“ occuper l'espace en utopie" (p. 185) comme l'écrit Brossard. C'est dans les zones libres de la cité intégrale et dans la traversée des frontières que s'opère le transfert utopique:

Ma vie privée est une carte sphérique d'influences et de points de rencontre, elle tourne autour de la langue comme hypothèse et filtre du quotidien fictif et théorique. Dans la conquête, je flaire un espace mental qui ne serait pas occupé par des descriptions, ici anecdote, là penchant a naturel • à refaire le même (musée, bungalow, Hilton); tendance à faire la femme. (p. 125)

D'où la nécessité d'élaborer le territoire imaginaire des femmes, "ma continent" pour reprendre l'expression de l'auteure dans Amantes ${ }^{16}$.

Dans sa préface à ce roman/poème que constitue Picture Theory, Louise Forsyth écrit que :

14 Ibid., p. 106.

15 Northrop Frye, "Varieties of Literary Utopias*, F. E. Manuel (dir.), Utopias and Utopian Thought, Boston/Cambridge, Houghton Mifflin Company/The Riverside Press, 1966, p. 49. Traduction: -[...] elles ne seraient pas des cités rationnelles pensées par la dialectique d'un philosophe: elles auraient leurs racines dans le corps comme dans l'esprit, dans l'inconscient autant que dans le conscient, dans les forêts et les déserts aussi bien que sur les autoroutes et dans les édifices. *

16 Nicole Brossard, Amantes, Montréal, Quinze, 1980, p. 103-109. 
on se tromperait si on voyait l'utopie de Nicole Brossard comme un lieu matêriel et stable qu'elle rêve de réaliser. Au contraire, cette utopie est dynamique, mobile et en mutation. Comme l'hologramme elle n'est que l'énergie intense: forme vitale et image cohérente. Elle est l'abstraction dynamique, l'énergie psychique, l'émotion concentrée. ${ }^{17}$

Elle est l'élan qui permet à l'énergie de cinq femmes "[t]raversières, urbaines radicales, lesbiennes " de prendre forme *comme l'électricité dans la structure de la matière elle-même" (p. 105). Cinq femmes, filles studieuses, explorant le dictionnaire, la sueur de la mer, entraînant avec elles "les mots tour à tour, spirale ignée, picture theory, une existence en ces termes" (p. 116). L'utopie brossardienne suppose l'abolition de toutes les utopies puisqu'il faudrait commencer par le mot "femme "; l'utopie serait alors *une fiction à partir de laquelle naîtrait le corps générique de celle qui pense", "celle par qui tout peut arriver" (p. 183). Pour Brossard, "Le corps générique deviendrait l'expression de la femme et la femme aurait des ailes au-dessus de tout, elle ferait signe. (p. 184). Parmi les trois possibles versions du livre de Michèle Vallée, le livre trois (La pensée) offre lui-même une structure tridimensionnelle. Dans la troisième partie ayant pour sous-titre SCREEN SKIN UTOPIA, la femme devient signe de l'utopie intégrale, le corps-écran d'un désir en mouvement, en traverses, en pentes. "Je disais, pense la narratrice, avec dans la bouche un goût de sel, à propos de l'utopie en commençant par le mot femme que l'utopie n'allait pas assurer notre insertion dans la réalité mais qu'un témoignage utopique de notre part pouvait stimuler une qualité d'émotion propice à notre insertion dans l'histoire ( p. 102).

Dans un tout autre registre textuel, face au Tapiz de la creación, il est évident que le visiteur se voit confronté à une utopie passéiste, un âge d'or perdu, un Éden qui ne fait que confirmer un pouvoir, un langage, une parole, celle d'un dieu qui tiendrait dans ses mains tous les fils de la toile (les fictions utopistes futuristes vont très souvent dans la même direction). Cependant, le lecteur de la tapisserie, en l'occurence Equis, peut aussi y déchiffrer un hologramme puisque les signes bougent, déplaçant le tissu et le sens di texte comme les vents représentés aux quatre

17 Louise H. Forsyth, - Préface , Picture Theory de Nicole Brossard, op. cit., p. 19-20. 
52

coins du tableau. Par contre, Adam et Ève y figurent dans les mêmes termes que dans la fiction biblique. Dans son article "Liberté et séquestration: de la condition de la femme en utopie", Adriana Sfragaro remarque que dans les utopies de justice, l'égalité des sexes est inexistante ou tout au moins à peine un élément troublant la conscience collective et individuelle, laquelle exige l'ordre et la stabilité. "Quand on ne peut éliminer la femme, écrit Sfragaro, on peut la sublimer (les utopies sont riches en honnêtes épouses, en bonnes mères, en citoyennes vertueuses); là où, théoriquement, on réhabilite la femme, pratiquement, on la neutralise" 18 .

Dans La nave de los locos, Peri Rossi retravaille la tapisserie médiévale à travers l'ironie et la parodie contenues dans les réponses qu'une quarantaine d'étudiants, sollicités de décrire Adam et Ėve et le paradis terrestre, fournissent à leur professeure, confirmant leur tendance à placer perpétuellement la femme dans un contexte social et politique réactionnaire, soit dans une utopie initiale faussée étant donné que la sortie est bloquée d'avance. Ainsi, deux étudiants, pour ne donner que quelques exemples plutôt amusants ou déroutants selon notre vision des choses, interprètent les rapports divin à la créature et masculin à la femme en ces mots : 1. "Dios sacó a Eva de una costilla de Adán porque el se aburría un poco y tenia ganas de tener a quien mandar"; ou encore dans les termes suivants: 2. "El (Adán) estava solo y no lo pasava muy bien porque no tenía con quien havlar pero cuando nació ella fue mucho peor ${ }^{19}$ (p. 157). D'autres conçoivent l'identité sexuelle de Adam et Eve à partir d'une vie quotidienne qu'ils recréent sur le mode de la répartition traditionnelle des tâches: 1. Adán se ocupaba de cazar fieras leones tigres y obejas. Eva limpiaba la casa y hasia las compras* (p. 159); 2. *Entonces Adán le dijo: Si quieres estudiar las ciencias del bien y del mal estúdialas, a mi no me importa, pero seguirás limpiando la casa y planchando, que es tu deber"; 3. "Adán estaba muy ocupado; no solo debía cuidar del paraíso que Dios le había

18 Adriana Sfragaro, - Liberté et séquestration: de la condition de la fermme en utopie., Carmelina Imbroscio (dir.), Requiem pour l'utopie?, Pise/Paris, Editrice Libreria Goliardica/Editions A.G. Nizet, 1986, p. 100.

19 Traduction: *1. Dieu a créé Ève à partir d'une côte d'Adam parce qu'il (Adam ou Dieu) s'ennuyait et parce qu'il avait envie de pouvoir donner des ordres à quelqu'un; 2. Il (Adam) était seul et s'ennuyait n'ayant personne avec qui parler mais à partir du moment de sa naissance (Ève) tout empira. - 
dado sino que además tenía que avastecer la vivienda. Ahora bien: además me parece que se encargaba de las relaciones públicas, porque él dialogaba directamente con Dios pero Eva no. (p. 160) 20 .

En résumé, l'ensemble de ces exemples dépeint le premier homme et la première femme en ces termes: Eve est perverse, frivole, curieuse, paresseuse, jalouse; une seule qualité, la beauté. Adam, quant à lui, est fort, courageux, honnête, travailleur, obéissant, responsable; un seul défaut, écouter les femmes. Bon nombre d'utopies (la cité idéale par exemple, Batman, Brazil, etc.) se construisent sur des stéréotypes et par conséquent sur la mort, note Giovanna Silvani dans son étude sur la femme et l'utopie (de More à Huxley), "both in the literary fiction and in the reality of our Western culture, in the present and in the past anchored to male values "niant par conséquent toute possibilité d'accomplissement au féminin ${ }^{21}$.

Dans le roman de Peri Rossi, la distopie ou l'utopie inversêe me semble venir en dernière instance de l'énigme qui hante Equis et que celui-ci réussit à résoudre à la toute fin du récit. À la question qui habite ses songes, à savoir " ¿cuál es el tributo mayor, el homenaje que un hombre puede hacer a la mujer que ama?. 22 , l'homme découvre que la réponse est "sa virilité" (p. 195-196). Faire abstraction de la virilité, de l'ego, ouvre la route à la découverte de nouveaux mondes et de nouvelles lectures. On s'embarque ainsi vers des terres étrangères et le concept de frontière fermé, limité ou inaccessible, se déplace considérablement. Les nouvelles utopies apparaissent donc susceptibles d'inclure l'altérité. Un passage révélateur précède

20 Traduction : : 1. Adam chassait des bêtes sauvages, des lions, des tigres et des moutons. Ève s'occupait du ménage et de l'épicerie; 2 . Alors Adam lui dit: $\mathrm{Si}$ tu veux étudier la science du bien et du mal, étudie-la, ça ne me fait rien, mais tu dois continuer de nettoyer la maison et de repasser, puisque c'est ton devoir; 3 . Adam était très occupé; non seulement devait-il prendre soin du paradis terrestre que Dieu lui avait donné mais il devait assurer la survie de sa famille. Et puis: de plus, il me semble qu'il s'occupait des relations publiques, car il dialoguait directement avec Dieu mais pas Eve.•

21 Giovanna Silvani, - Woman in Utopia from More to Huxley *, ibid., p. 152. Traduction: : [...] à la fois dans la fiction littéraire et dans la réalité de notre culture occidentale, dans le présent et le passé amarrés aux valeurs patriarcales.

22 Traduction: $\cdot[. .$.$] quel est la plus grande louange, le plus grand hommage$ qu'un homme puisse rendre à la femme qu'il aime?. 
54

d'ailleurs cet épisode au cours duquel Equis fait le constat de cette nécessité: il s'agit d'une scène érotique dans laquelle Marlene Dietrich et Dolores del Río (représentées par des femmes, des hommes ou des travestis, l'ambiguïté demeure) jouent au milieu d'une salle en délire la scène primordiale de l'inversion et de la séduction des sexes. Le texte abolit ou déconstruit de la sorte la création de l'homme, la rendant inutile pour la procréation et la suite du monde, renversant la fonction première du musée contenue dans la tapisserie, dans toute ouvre d'art.

Les utopies proposées par Peri Rossi et Brossard supposent une transgression permanente; nouvelles perspectives instaurant par ailleurs des formes de contre-utopie ou d'anti-utopie qui ne situent plus le discours utopique et fictif uniquement dans le nonlieu, l'ailleurs imaginaire, l'inconnu. Marina Leslie signale à ce propos que "the map (map of Utopia) registers both the gap (between here and there, then and now) and the critical distance which makes the gap legible, comprehensible, in short meaningful". Reprenant les termes de Claudio Guillén, elle attire notre attention sur le fait que la perspective conditionne toujours l'examen et le regard (retour au musée par la porte d'à côté); lire l'Utopie implique donc de se situer dans l'espace, face à la frontière ${ }^{23}$. Pour cette raison, il faut conclure avec l'auteure que la seule utopie véritablement authentique (anti-idéologique) serait celle qui affirme son désir de ne persuader personne. Autrement, l'utopie qui chercherait à déranger la pensée et l'histoire serait automatiquement récupérée par les formes idéologiques réactionnaires.

La pensée féministe risque à tout moment d'être récupérée par le discours historique et politique qu'elle vient délibérément chambarder. Mais les nouvelles utopies féministes dont il est question ici cherchent d'abord et avant tout à renverser des idéologies véhiculées par un système patriarcal et capitaliste clos. Brossard propose de lire l'utopie en termes d'énergie et de mouvement ${ }^{24}$. Finalement, l'utopie se pense chez ces écrivaines

23 Marina Leslie, -Mapping Out Ideology: The Case of Utopia ", RS/SI, vol. XII, $n^{\text {os }} 1-2,1992$, p. 90. Traduction: $-[\ldots]$ la carte d'Utopie enregistre simultanément l'espace (entre ici et là, alors et maintenant) et la distance critique qui rend l'espace lisible, compréhensible, bref signifiant .

24 Jameson suggère de penser le discours d'Utopie en termes de neutralité et de neutralisation, c'est-à-dire en mettant l'accent sur le progrès, l'energeia, 
comme un projet, celui d'inventer un monde dans lequel on pourrait imaginer d'abolir ou d'ouvrir les frontières, de refaire les cartes, d'explorer les mentalités et les cultures de l'autre, de transformer le je/sujet silencieux des femmes en lieux de paroles, d'instaurer des formes de migrations permanentes et signifiantes.

l'énonciation, la productivité, et non en fonction d'une représentation traditionnelle d'un modèle social idéal. Voir Fredric Jameson, Naturalization and the Production of Utopian Discourse *, Diacritics, n 7, 1977, p. 6. 